ESAIM: PROCEEDINGS AND SURVEYS, June 2017, Vol. 56, p. 139-143

S. Crépey, M. Jeanblanc and A. Nikeghbali Editors

\title{
GROSSISSEMENTS DE FILTRATIONS : GROSSISSEMENTS INITIAUX ET PROGRESSIFS
}

\author{
MARC YOR
}

\begin{abstract}
We reproduce here two texts from Marc Yor, parts of his "testament scientifique" in relation with enlargement of filtration. The full version of these texts can be found on http://www.mathevry.cnrs.fr/pmf/marc_yor/publication?.
\end{abstract}

\section{TEXTE ÉCRIT EN 2006}

On trouvera ci-dessous vingt thèmes qui m'ont occupé, ainsi que mes articles correspondants, entre 1973 et 2003. Pour chacun de ces thèmes, j'ai présenté - de façon informelle - le théorème principal sous la forme Th N ; quelquefois, j'ai ajouté un petit commentaire, puis une bibliographie complémentaire, composée d'articles liés étroitement au thème correspondant.

$[\ldots]$

\section{Thème 4 : Grossissements de filtrations}

(1) Grossissement d'une filtration, et semi-martingales : théorèmes généraux. Sém. Probas XII (Strasbourg), Lect. Notes in Maths. 649, Springer (1978), p. 61-69.

(2) Grossissement d'une filtration, et semi-martingales : formules explicites. (avec T. Jeulin). Sém. Probas XII (Strasbourg), Lect. Notes in Maths. 649, Springer (1978), p. 78-97.

(3) Nouveaux résultats sur le grossissement des tribus. (avec T. Jeulin). Ann. Scient. E.N.S., 4ème série, t. $11,(1978)$, p. $429-433$.

(4) Grossissement de filtrations et absolue continuité de noyaux. Grossissement de filtrations : exemples et applications. Lect. Notes in Maths. 1118, Springer (1985), p. 7-14

(5) Inégalités de martingales continues arrêtées à un temps quelconque. Grossissement de filtrations : exemples et applications. Lect. Notes in Maths. 1118, Springer (1985). I : Théorèmes généraux, p. 110-146, II : Le rôle de certains espaces BMO, p. 147-171.

(6) Entropie d'une partition, et grossissement initial d'une filtration. Grossissement de filtrations : exemples et applications. Lect. Notes in Maths. 1118, Springer (1985), p. 45-58.

Theorem 1.1. Une filtration étant donnée, et $L$ une variable aléatoire à valeurs dans $\mathbb{R}^{+}$(c'est-à-dire un temps, qui n'est pas a priori un temps d'arrêt de la filtration), on considère la plus petite filtration contenant la filtration d'origine, et faisant de ce temps $L$ un temps d'arrêt. Alors, toute martingale dans la filtration d'origine, arrêtée en L, reste une semi-martingale dans la nouvelle filtration. 
Commentaire 1: Un des points de départ de la théorie du grossissement de filtration; cf, LNM 833 (T. Jeulin; 1980) et 1118 (eds. T. Jeulin, M. Yor; 1985).

Bibliographie : M.T. Barlow : Study of a filtration expanded to include an honest time. ZfW 44, 1978, p. 307-324.

Commentaire 2: Les développements autour des grossissements de filtration ont été motivés par les questions que se posaient à ce sujet, indépendamment les uns des autres, K. Itô (1976) : Extension of Stochastic integrals, D. Williams, qui a soumis le problème à M.T. Barlow (76-77), et P.A. Meyer (également en 77) qui m'a soumis le problème de grossissement progressif (ie : faire d'un temps aléatoire un temps d'arrêt). Publication d'un chapitre entier (le dernier) sur le grossissement de filtration dans la nouvelle édition de Ph. Protter (2003). Les questions d'asymétrie d'information dans les marchés financiers (ou encore : problèmes d'initiés) ont motivé, dans les dix dernières années, beaucoup de développements sur le grossissement initial de filtrations. Des développements analogues avec le grossissement progressif devraient avoir lieu à partir de problèmes de risques de défaut.

\section{TEXT WRITTEN IN 2014}

(a) La notion de martingale est relative... De façon précise, lorsque l'on dit que le processus $\left(M_{t}, t \geq 0\right)$ est une martingale, on sous-entend : par rapport à un espace de probabilité filtré $\left(\Omega, \mathcal{F},\left(\mathcal{F}_{t}\right)_{t \geq 0}, P\right)$ pour lequel :

$$
E\left[M_{t} \mid \mathcal{F}_{s}\right]=M_{s} \quad(s \leq t),
$$

et $\left(M_{t}\right)$ est $\left(\mathcal{F}_{t}\right)$ adapté. Lorsque l'on ne précise pas la filtration $\left(\mathcal{F}_{t}\right)$, on peut penser raisonnablement que c'est la filtration naturelle de $\left(M_{t}\right)$ qui est en jeu, i.e :

$$
\mathcal{M}_{t}=\sigma\left\{M_{s}, s \leq t\right\} .
$$

Remarquons que, si $\left(\mathcal{J}_{t}\right)$ est n'importe quelle filtration intermédiaire entre $\left(\mathcal{M}_{t}\right)$ et $\left(\mathcal{F}_{t}\right)$, alors la $\left(\mathcal{F}_{t}\right)$ martingale $\left(M_{t}\right)$ est une $\left(\mathcal{J}_{t}\right)$ martingale. Il n'en est pas de même lorsque l'on remplace $\left(\mathcal{J}_{t}\right)$ par une surfiltration $\left(\mathcal{S}_{t}\right)$ de $\left(\mathcal{F}_{t}\right)$. En fait, il est assez rare que $\left(M_{t}\right)$ soit encore une $\left(\mathcal{S}_{t}\right)$ martingale.

Dans la suite, plutôt que de considérer ces questions de façon individuelle, nous allons les étudier pour toutes les $\left(\mathcal{F}_{t}\right)$ martingales à la fois. Soit donc, sur un espace de probabilité $(\Omega, \mathcal{F}, P)$, deux filtrations $\left(\mathcal{F}_{t}\right)_{t \geq 0}$ et $\left(\mathcal{S}_{t}\right)_{t \geq 0}$ telles que, pour tout $t, \mathcal{F}_{t} \subset \mathcal{S}_{t} \subset \mathcal{F}$.

Essayons de répondre aux questions suivantes :

Question 1: A quelle condition est-ce que toute $\left(\mathcal{F}_{t}\right)$ martingale est une $\left(\mathcal{S}_{t}\right)$ martingale ? On dit alors que $\left(\mathcal{F}_{t}\right)$ est immergée dans $\left(\mathcal{S}_{t}\right)$.

La réponse à cette question, donnée par le théorème 2.1, est assez "rigide" et amène à se poser une seconde question, un peu plus "souple".

Question 2: A quelle condition est-ce que toute $\left(\mathcal{F}_{t}\right)$ martingale est une $\left(\mathcal{S}_{t}\right)$ semimartingale ? On dit alors que $\left(\mathcal{F}_{t}\right)$ est semi-immergée dans $\left(\mathcal{S}_{t}\right)$.

On peut estimer que, s'il en est ainsi, c'est que la "surinformation" apportée par $\left(\mathcal{S}_{t}\right)$ au-dessus de $\left(\mathcal{F}_{t}\right)$ est "raisonnable".

La question est donc, une filtration $\left(\mathcal{F}_{t}\right)$ étant donnée, de déterminer les "surinformations raisonnables" audessus de $\left(\mathcal{F}_{t}\right)$. De façon un peu étonnante, il n'y a pas eu d'étude vraiment générale de ce problème, souvent appelé : problème de grossissement de filtration. Toutefois, deux cas ont été étudiés de façon très systématique : - celui du grossissement initial de $\left(\mathcal{F}_{t}\right)$, à l'aide d'une variable aléatoire $X$; c'est-à-dire que $: \mathcal{S}_{t}=\mathcal{F}_{t} \vee \sigma(X)$; - celui du grossissement progressif de $\left(\mathcal{F}_{t}\right)$, consistant à faire d'une variable aléatoire $\tau \geq 0$ (: un temps aléatoire) un temps d'arrêt; ainsi, si $\mathcal{S}_{t}^{\tau}=\mathcal{F}_{t} \vee \sigma(\tau \leq s ; s \leq t)$, est-ce que toute $\left(\mathcal{F}_{t}\right)$ martingale reste une semimartingale? Le théorème 2.3 (plus bas) répond partiellement à cette question. 
Theorem 2.1. Toute $\left(\mathcal{F}_{t}\right)$ martingale est une $\left(\mathcal{S}_{t}\right)$ martingale, si, et seulement si, pour tout $t$ fixé, $\mathcal{F}_{\infty}$ et $\mathcal{S}_{t}$ sont conditionnellement indépendantes sachant $\mathcal{F}_{t}$.

Corollary 2.2. Si pour tout, $\mathcal{F}_{t} \subset \mathcal{S}_{t} \subset \mathcal{F}_{\infty}$, et si $\left(\mathcal{F}_{t}\right)$ est immergée dans $\left(\mathcal{S}_{t}\right)$, alors la filtration $\left(\mathcal{S}_{t}\right)$ est identique $\grave{a}\left(\mathcal{F}_{t}\right)$.

Démonstration : D'après le théorème 2.1, s'il y a immersion, on doit donc ainsi : $\forall F_{\infty} \in \mathcal{F}_{\infty}, E\left[1_{F_{\infty}} \mid \mathcal{S}_{t}\right]=$ $E\left[1_{F_{\infty}} \mid \mathcal{F}_{t}\right]$. Ceci s'applique en particulier à tout ensemble $S_{t} \in \mathcal{S}_{t}$, donc : $1_{S_{t}}=E\left[1_{S_{t}} \mid \mathcal{F}_{t}\right]$ est $\left(\mathcal{F}_{t}\right)$ mesurable. Ainsi, sous la seule hypothèse : $\mathcal{F}_{t} \varsubsetneqq \mathcal{S}_{t} \subseteq \mathcal{F}_{\infty}$, il existe au moins une $\left(\mathcal{F}_{t}\right)$ martingale qui n'est pas une $\mathcal{S}_{t}$ martingale, et la question 2 apparaît donc très naturelle.

(b) Faisons quelques préparations pour énoncer le théorème 2.3 : on suppose que toute $\left(\mathcal{F}_{t}\right)$ martingale est continue (hypothèse $(\mathrm{C})$ ), et, d'autre part, que $\tau$ est la fin d'un ensemble $\left(\mathcal{F}_{t}\right)$ prévisible, c'est-à-dire :

$$
\tau=\sup \{t:(t, \omega) \in \Gamma\}
$$

où $\Gamma$ est un ensemble $\left(\mathcal{F}_{t}\right)$ prévisible. J. Azéma a associé à $\tau$ la surmartingale :

$$
Z_{t}=P\left(\tau>t \mid \mathcal{F}_{t}\right)
$$

On suppose que pour tout $\left(\mathcal{F}_{t}\right)$ temps d'arrêt $T$ on a : $P(\tau=T)=0$. On note $(A)$ cette hypothèse.

On a alors le :

Theorem 2.3. Sous les hypothèses $(C)$ et $(A)$, toute $\left(\mathcal{F}_{t}\right)$ martingale $\left(M_{t}\right)$ est une $\left(\mathcal{S}_{t}^{\tau}\right)$ semimartingale, dont la décomposition canonique est :

$$
M_{t}=\widetilde{M}_{t}+\int_{0}^{\tau \wedge t} \frac{d<M, Z>_{s}}{Z_{s}}+\int_{\tau}^{t} \frac{d<M, 1-Z>_{s}}{1-Z_{s}}
$$

où $\left(\widetilde{M}_{t}\right)$ est une $\left(\mathcal{S}_{t}^{\tau}\right)$ martingale locale.

(c) Commentaires : Voici quelques illustrations des théorèmes 2.1 et 2.3.

(c.1) A propos du théorème 2.1.

- Si $\left(B_{t}, t \geq 0\right)$ est un mouvement brownien unidimensionnel, et $B_{t}^{(n)}=\left(B_{t}, \beta_{t}^{(2)}, \ldots, \beta_{t}^{(n)}\right)$ un mouvement brownien $n$-dimensionnel, dont la première composante est $\left(B_{t}\right)$, alors toute $\left(\mathcal{B}_{t}=\sigma\left\{B_{s}, s \leq t\right\}, t \geq 0\right)$ martingale est une $\left(\mathcal{B}_{t}^{(n)}\right)$ martingale.

Cela découle aisément de ce que : $\mathcal{B}_{t}^{(n)}=\mathcal{B}_{t} \vee \mathcal{C}_{t}$, o $\tilde{A} z \mathcal{C}_{t}=\sigma\left\{\beta_{s}^{(2)}, \ldots, \beta_{s}^{(n)} ; s \leq t\right\}$, est indépendante de $\mathcal{B}_{t}$.

- Voici quelques exemples un peu moins évidents

(i) $\left(B_{t}, t \geq 0\right)$ désignant toujours un mouvement brownien unidimensionnel, soit $\beta_{t}=\int_{0}^{t} \operatorname{sgn}\left(B_{s}\right) d B_{s}, t \geq 0$. Alors, la filtration naturelle de $\beta$, soit $\left(\widetilde{\mathcal{B}}_{t}\right)$, est celle de la valeur absolue de $B$, ce qui découle assez facilement de la formule de Tanaka :

$$
\left|B_{t}\right|=\beta_{t}+L_{t}, t \geq 0,(\text { cf. Thème } 2)
$$

Toute $\left(\widetilde{\mathcal{B}}_{t}\right)$ martingale étant une intégrale stochastique par rapport à $\left(d \beta_{s}\right)$, est une $\left(\mathcal{B}_{t}\right)$ martingale, 
(ii) Pour $n>1$, notons $R_{t}=\left|B_{t}^{(n)}\right|$ (le processus de Bessel de dimension $n$, dont il sera question dans le Thème 5). Sa filtration naturelle $\left(\mathcal{R}_{t}\right)$ est celle du mouvement brownien $\left(\beta_{t}^{(n)}\right)$ tel que :

$$
\mathcal{R}_{t}=\beta_{t}^{(n)}+\frac{n-1}{2} \int_{0}^{t} \frac{d s}{R_{s}}
$$

On a, de plus :

$$
\beta_{t}^{(n)}=\sum_{i=1}^{n} \int_{0}^{t} \frac{B_{s}^{(i)} d B_{s}^{(i)}}{R_{s}}
$$

Un argument semblable à celui utilisé en (i) montre que toute $\left(\mathcal{R}_{t}\right)$ martingale est une $\left(\mathcal{B}_{t}^{(n)}\right)$ martingale. - Voici enfin un résultat "négatif" qui peut sembler un peu troublant compte tenu des paragraphes (i) et (ii) ci-dessus.

Proposition 2.4. Soit $\left(B_{t}\right)$ mouvement brownien unidimensionnel. Il n'existe pas de surfiltration $\left(\mathcal{S}_{t}\right)$ de $\left(\mathcal{B}_{t}\right)$ telle que : $\mathcal{B}_{t} \subset \mathcal{S}_{t} \subset \mathcal{B}_{\infty}$, pour laquelle $\left(B_{t}\right)$ soit une $\left(\mathcal{S}_{t}\right)$ martingale.

Première démonstration : Il suffit d'appliquer le Corollaire 2.2, car si $\left(B_{t}\right)$ est une $\left(\mathcal{S}_{t}\right)$ martingale, alors toute $\left(\mathcal{B}_{t}\right)$ martingale (qui est une intégrale stochastique par rapport à $\left(d B_{t}\right)$ ) est une $\left(\mathcal{S}_{t}\right)$ martingale.

Démonstration : (elle reprend, plus doucement!, les arguments de la première démonstration) Soit donc $\left(\mathcal{S}_{t}\right)$ surfiltration de $\left(\mathcal{B}_{t}\right)$ satisfaisant les conditions de l'énoncé.

Fixons $t_{o}>0$, et considérons $\Gamma_{t_{o}} \in \mathcal{S}_{t_{o}}$.

Par hypothèse, on a :

$$
E\left[1_{\Gamma_{t_{o}}}\left(B_{t}-B_{t_{o}}\right)\right]=0, \text { pour tout } t \geq t_{o},
$$

ce qui implique plus généralement que pour tout processus $\left(H_{u}\right),\left(\mathcal{B}_{u}\right)$ prévisible, tel que :

$$
E\left[\int_{t_{o}}^{\infty} H_{u}^{2} d u\right]<\infty
$$

on ait :

$$
E\left[1_{\Gamma_{t_{o}}} \int_{t_{o}}^{\infty} H_{u} d B_{u}\right]=0 .
$$

Or, d'après la propriété de représentation prévisible pour le mouvement brownien $\left(B_{t}\right)$ (dans sa propre filtration $\left(\mathcal{B}_{t}\right)$ ), on a, en utilisant l'hypothèse que : $\Gamma_{t_{o}} \in \mathcal{B}_{\infty}$ :

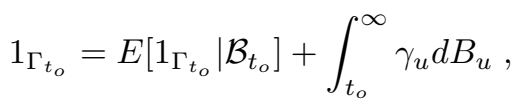

pour un certain processus $\left(\mathcal{B}_{u}\right)$ prévisible $\left(\gamma_{u}\right)$ tel que $E\left[\int_{t_{o}}^{\infty} \gamma_{u}^{2} d u\right]<\infty$. Prenant $H=\gamma$ en (3), on obtient : $\int_{t_{o}}^{\infty} \gamma_{u} d B_{u}=0$, donc d'après (4),

$$
1_{\Gamma_{t_{o}}}=E\left[1_{\Gamma_{t_{o}}} \mid \mathcal{B}_{t_{o}}\right]
$$

En conséquence, $\left(\mathcal{S}_{t}\right)$ est identiquement égale à $\left(\mathcal{B}_{t}\right)$.

Remarques : a) La Proposition 2.4 ne contredit pas les exemples de surfiltrations présentés en (i) et (ii) ci-dessus, car dans ces deux cas :

$$
\widetilde{\mathcal{B}}_{\infty} \varsubsetneqq \mathcal{B}_{\infty} \text { et } \mathcal{R}_{\infty} \varsubsetneqq \mathcal{B}_{\infty}^{(n)}
$$


b) Voici maintenant un exemple de situation très différente de celle de la Proposition 2.4 :

considérons : $A_{t}=\int_{0}^{t}\left(X_{s} d Y_{s}-Y_{s} d X_{s}\right), t \geq 0$, le processus de l'aire stochastique (de Lévy) du mouvement brownien plan $\left(X_{t}+i Y_{t}, t \geq 0\right)$. Notons $\left(\mathcal{A}_{t}\right)_{t \geq 0}$ la filtration naturelle du processus $\left(A_{t}\right)_{t \geq 0}$. C'est la filtration d'un mouvement brownien 2-dimensionnel :

$$
\left(\gamma_{t}=\int_{0}^{t} \frac{X_{s} d Y_{s}-Y_{s} d X_{s}}{R_{s}}, \beta_{t}=R_{t}-\frac{1}{2} \int_{0}^{t} \frac{d s}{R_{s}} ; t \geq 0\right)
$$

o $\tilde{\mathrm{A} z ́} R_{t}=\left(X_{t}^{2}+Y_{t}^{2}\right)^{1 / 2}$, et il n'est pas difficile de montrer que $\left(A_{t}\right)$ est encore une martingale dans la surfiltration $\left(\widetilde{\mathcal{A}}_{t}\right)$ de $\left(\mathcal{A}_{t}\right)$, définie par : $\widetilde{\mathcal{A}}_{t}=\mathcal{A}_{t} \vee \sigma\left\{\beta_{s} ; s \geq 0\right\}$, et on a bien :

$$
\mathcal{A}_{t} \subset \widetilde{\mathcal{A}}_{t} \subset \mathcal{A}_{\infty}
$$

(c.2) A propos du théorème 2.3

Un certain nombre d'exemples de fins d'ensembles $\left(\mathcal{F}_{t}\right)$ prévisibles $\tau$, satisfaisant les hypothèses $(\mathrm{C})$ et $(\mathrm{A})$, ont été présentés dans la Monographie : Mansuy-Yor [B.7].

\section{REFERENCES}

$[\mathrm{A}]$

[A.1] K. Itô. Extension of stochastic integrals. In Proceedings of the International Symposium on Stochastic Differential Equations (Res. Inst. Math. Sci., Kyoto Univ., Kyoto, 1976), pages 95-109, Wiley 1978.

[A.2] M. Barlow Study of a filtration expanded to include an honest time. Z. Wahrsch. Verw., 44(4), pages 307-323, 1978. [B]

[B.1] M. Yor. Grossissement d'une filtration et semi-martingales: théorèmes généraux. In Séminaire de Probabilités, XII (Univ. Strasbourg, Strasbourg, 1976/1977), volume 649 of Lecture Notes in Math., pages 61-69. Springer, Berlin, 1978.

[B.2] T. Jeulin et M. Yor. Grossissement d'une filtration et semi-martingales: formules explicites. In Séminaire de Probabilités, XII (Univ. Strasbourg, Strasbourg, 1976/1977), volume 649 of Lecture Notes in Math., pages 78-97. Springer, Berlin, 1978.

[B.3] T. Jeulin et M. Yor. Nouveaux résultats sur le grossissement des tribus. Ann. Sci. École Norm. Sup. (4), 11(3):429-443, 1978.

[B.4] M. Yor. Grossissement de filtrations et absolue continuité de noyaux. In Grossissement de filtrations: exemples et applications, volume 1118 of Lecture Notes in Math., page 6. Springer, Berlin, 1985.

[B.5] M. Yor. Inégalités de martingales continues arrêtées à un temps quelconque. 1 : Théorèmes généraux, 2 : Le rôle de certains espaces BMO. In Grossissement de filtrations: exemples et applications, volume 1118 of Lecture Notes in Math., pages 11-14. Springer, Berlin, 1985.

[B.6] M. Yor. Entropie d'une partition, et grossissement initial d'une filtration. In Grossissement de filtrations: exemples et applications, volume 1118 of Lecture Notes in Math., page 45. Springer, Berlin, 1985.

[B.7] R. Mansuy et M. Yor. Random times and enlargements of filtrations in a Brownian setting. Lecture Notes in Math. 1873, Springer, 2006. 